\title{
Numerical Solutions of Time Dependent Partial Differential Equations Using Weighted Residual Method With Piecewise Polynomials
}

\author{
Muntasir Alam and Md. Shafiqul Islam* \\ Department of Applied Mathematics, Dhaka University, Dhaka-1000, Bangladesh
}

(Received: 14 September 2017; Accepted: 27 December 2018)

\begin{abstract}
We use Galerkin weighted residual (GWR) method to solve one dimensional heat and wave equations as initial and boundary value problems (IBVPs) numerically. Three special types of piecewise polynomials namely: Bernstein, Bernoulli and Legendre polynomials are used as basis functions to solve these IBVPs. A few examples are tested by the proposed method and then the results are compared with the solutions found in other existing methods. The numerical results obtained in this paper are in good agreement with the exact solutions.
\end{abstract}

Keywords: Diffusion equation, wave equation, Galerkin Weighted Residual method, Bernstein, Legendre and Bernoulli Polynomials.

\section{Introduction}

One of the main interests for studying this topic is to generate the numerical solutions in a much efficient way with significant level of accuracy. In this paper, we initially develop a rigorous formulation on Galerkin Weighted Residual Method (WRM) so that the $\operatorname{IBVPs}^{1,2,3}$ can be solved easily with approximate solutions which converge rapidly to exact solutions. For this, we give a details Galerkin formulation for parabolic and hyperbolic IBVPS subsequently using the three types of piecewise polynomials such as Bernstein ${ }^{7}$, Bernoulli ${ }^{6}$ and Legendre ${ }^{7}$ polynomials.

\section{Formulation of One Dimensional Diffusion Equation}

In this section, the Bernstein, Bernoulli and Legendre polynomials are chosen in such a way that they satisfy the given Dirichlet boundary conditions.

In the formulation ${ }^{3}$ the unknown function $u(x, t)$ is treated as a function of both space and time. We consider the partial differential equation of the form:

$\mu(x) \frac{\partial u(x, t)}{\partial t}-\frac{\partial}{\partial x}\left(\alpha(x) \frac{\partial u(x, t)}{\partial x}\right)+\beta(x) u(x, t)=$ $f(x, t), a<x<b ; t>t_{0}$

with boundary conditions:

at $x=a\left(t>t_{0}\right)$

$u(a, t)=u_{a}(t),\left(-\alpha(x) \frac{\partial u(x, t)}{\partial x}\right)_{a}=\tau_{a}(t) ;$

at $x=b\left(t>t_{0}\right)$

$u(b, t)=u_{b}(t),\left(-\alpha(x) \frac{\partial u(x, t)}{\partial x}\right)_{b}=\tau_{b}(t)$

along with the initial condition

at $t=t_{0}(a<x<b) ; \quad u\left(x, t_{0}\right)=u_{0}(x)$.

Let us take the trial solution of Eq. (1) be of the form:

$\tilde{u}(x, t)=\sum_{j=1}^{n} a_{j}(t) \varphi_{j}(x)$

where, $\varphi_{j}(x)$ denotes the trial functions in space variable of the type - Bernstein, Bernoulli or Legendre polynomials, and the parameters $a_{j}(t)$ be functions of time only.
Then the Galerkin Weighted Residual equation for the interval $a<x<b$ may be written as

$$
\begin{array}{r}
\int_{a}^{b}\left[\mu(x) \frac{\partial \tilde{u}(x, t)}{\partial t}-\frac{\partial}{\partial x}\left(\alpha(x) \frac{\partial \tilde{u}(x, t)}{\partial x}\right)+\beta(x) \tilde{u}(x, t)\right. \\
-f(x, t)] \varphi_{i}(x) d x=0, i=1,2, \ldots, n ;
\end{array}
$$

Using the conditions we get the simplified form the of equation as

$\sum_{j=1}^{n} C_{i, j} \frac{d a_{j}(t)}{d t}+\sum_{j=1}^{n} K_{i, j} a_{j}(t)=F_{i}(t), i=1,2,3, \ldots, n$

which can be expressed in usual matrix notation as

$[C]\left\{\frac{d a(t)}{d t}\right\}+[K]\{a(t)\}=\{F(t)\}$

where,

$$
\begin{aligned}
& {[C]=C_{i, j}=\int_{a}^{b} \varphi_{i}(x) \mu(x) \varphi_{j}(x) d x} \\
& \begin{aligned}
{[K]=K_{i, j}=\int_{a}^{b} \frac{d \varphi_{i}(x)}{d x} \alpha(x) \frac{d \varphi_{j}(x)}{d x} d x } \\
\quad+\int_{a}^{b} \varphi_{i}(x) \beta(x) \varphi_{j}(x) d x \\
F_{i}=\int_{a}^{b} f(x, t) \varphi_{i}(x) d x-\left[\left(-\alpha(x) \frac{\partial \tilde{u}(x, t)}{\partial x}\right) \varphi_{i}(x)\right]_{a}^{b}
\end{aligned}
\end{aligned}
$$

In the above notation $C_{i, j}$ and $K_{i, j}$ are known as the heat capacity matrix and stiffness matrix respectively, whereas $F_{i}$ is called the load matrix. Now all it need to solve Eq. (3) using the given conditions

Now the system of differential equations (3) will be treated analytically by decomposing the general solution $a(t)$ into a homogeneous solution $a_{h}$ and a particular solution $a_{p}$ according to

$a(t)=a_{h}(t)+a_{p}(t)$

Using Eq. (4) we get our desired solution from Eq. (2). 


\section{Numerical Examples}

In this section, we consider two examples to verify the proposed formulations described in section II. All the computations are performed by using MATLAB.

\section{Example 1.}

Consider the one-dimensional heat conduction problem ${ }^{3}$

$$
u_{t}=u_{x x} ; 0<x<1 ; t>0
$$

with boundary conditions: $u(0, t)=u(1, t)=0$

and initial condition: $u(x, 0)=u_{0}(x)=x(1-x)$.

The exact solution is:

$u(x, t)=\sum_{k=1}^{\infty} \frac{8}{(2 k-1)^{3} \pi^{3}} e^{-t \pi^{2}(2 k-1)^{2}} \sin ((2 k-1) \pi x)$.

Table 1. Comparison of results (at $t=0.1$ )

\begin{tabular}{|c|c|c|c|c|}
\hline \multirow{2}{*}{$x$} & \multirow{2}{*}{$\begin{array}{c}\text { Exact } \\
\text { Solutions }\end{array}$} & \multicolumn{3}{|c|}{ Relative Error } \\
\cline { 3 - 5 } & & Bernstein & Bernoulli & Legendre \\
\cline { 3 - 5 } 0.0 & 0.0000000000 & 0.0000000000 & 0.0000000000 & 0.0000000000 \\
\hline 0.1 & 0.0297171352 & $1.1414 \times 10^{-1}$ & $1.1414 \times 10^{-1}$ & $1.1414 \times 10^{-1}$ \\
\hline 0.2 & 0.0565245706 & $4.1329 \times 10^{-2}$ & $4.1329 \times 10^{-2}$ & $4.1329 \times 10^{-2}$ \\
\hline 0.3 & 0.0777980709 & $6.9845 \times 10^{-3}$ & $6.9845 \times 10^{-3}$ & $6.9845 \times 10^{-3}$ \\
\hline 0.4 & 0.0914558562 & $3.4604 \times 10^{-2}$ & $3.4604 \times 10^{-2}$ & $3.4604 \times 10^{-2}$ \\
\hline 0.5 & 0.0961618714 & $4.3593 \times 10^{-2}$ & $4.3593 \times 10^{-2}$ & $4.3593 \times 10^{-2}$ \\
\hline & & \multicolumn{3}{|c|}{ Parameters used $n=3$} \\
\hline 0.0 & 0.0000000000 & 0.0000000000 & 0.0000000000 & 0.0000000000 \\
\hline 0.1 & 0.0297171352 & $3.9487 \times 10^{-4}$ & $5.5798 \times 10^{-4}$ & $5.7548 \times 10^{-4}$ \\
\hline 0.2 & 0.0565245706 & $1.3481 \times 10^{-3}$ & $1.3547 \times 10^{-3}$ & $1.3537 \times 10^{-3}$ \\
\hline 0.3 & 0.0777980709 & $6.9431 \times 10^{-4}$ & $6.8878 \times 10^{-4}$ & $6.9852 \times 10^{-4}$ \\
\hline 0.4 & 0.0914558562 & $4.2039 \times 10^{-4}$ & $4.3269 \times 10^{-4}$ & $4.1667 \times 10^{-4}$ \\
\hline 0.5 & 0.0961618714 & $9.0404 \times 10^{-4}$ & $9.1851 \times 10^{-4}$ & $8.9999 \times 10^{-4}$ \\
\hline & & \multicolumn{3}{|c|}{ Parameters used $n=4$} \\
\hline 0.0 & 0.0000000000 & 0.0000000000 & 0.0000000000 & 0.0000000000 \\
\hline 0.1 & 0.0297171352 & $5.6963 \times 10^{-4}$ & $7.0658 \times 10^{-4}$ & $1.1682 \times 10^{-2}$ \\
\hline 0.2 & 0.0565245706 & $1.3461 \times 10^{-4}$ & $1.2054 \times 10^{-3}$ & $9.7982 \times 10^{-3}$ \\
\hline 0.3 & 0.0777980709 & $6.8201 \times 10^{-4}$ & $5.3931 \times 10^{-4}$ & $1.0451 \times 10^{-2}$ \\
\hline 0.4 & 0.0914558562 & $4.3841 \times 10^{-4}$ & $5.8213 \times 10^{-4}$ & $1.1559 \times 10^{-2}$ \\
\hline 0.5 & 0.0961618714 & $9.2387 \times 10^{-4}$ & $1.0679 \times 10^{-3}$ & $1.2041 \times 10^{-2}$ \\
\hline & & \multicolumn{3}{|c|}{ Parameters used $n=5$} \\
\hline 0.0 & 0.0000000000 & 0.0000000000 & 0.0000000000 & 0.0000000000 \\
\hline 0.1 & 0.0297171352 & $6.2622 \times 10^{-5}$ & $3.1212 \times 10^{-5}$ & $9.5535 \times 10^{-3}$ \\
\hline 0.2 & 0.0565245706 & $2.9596 \times 10^{-5}$ & $1.3954 \times 10^{-5}$ & $9.5992 \times 10^{-3}$ \\
\hline 0.3 & 0.0777980709 & $3.6327 \times 10^{-6}$ & $2.0537 \times 10^{-5}$ & $9.6059 \times 10^{-3}$ \\
\hline 0.4 & 0.0914558562 & $5.6782 \times 10^{-6}$ & $3.4464 \times 10^{-6}$ & $9.5818 \times 10^{-3}$ \\
\hline 0.5 & 0.0961618714 & $9.3885 \times 10^{-6}$ & $1.7606 \times 10^{-6}$ & $9.5675 \times 10^{-3}$ \\
\hline & & \multicolumn{3}{|c}{} \\
\hline
\end{tabular}

\section{Tabular Analysis of Solutions}

In this part, we show our results in Table 1. It is evident that Bernstein polynomials give the best accuracy with the minimum number of polynomials. The accuracy level is quite high when we use more parameters. It is also seen that Bernoulli polynomials are also very efficient for faster converge whereas the Legendre polynomials provide less accuracy than the other two. For $n=5$, the polynomials are depicted in Fig.1. Similarly, we plot the graphs of error functions using Bernstein, Bernoulli and Legendre polynomials in Fig. 2a-2c, respectively, for different $n$.

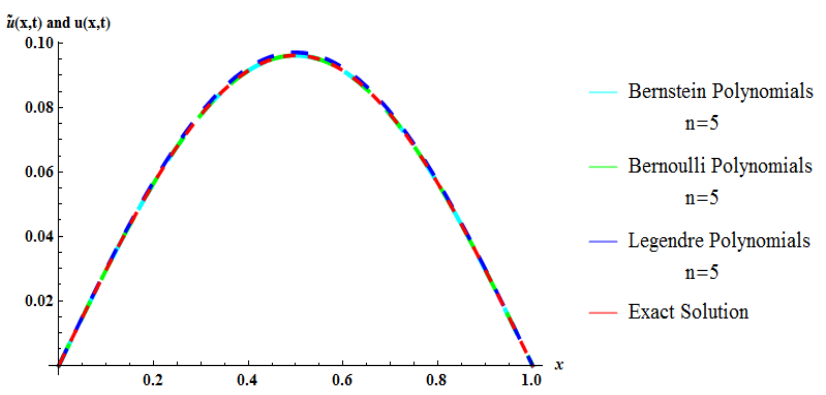

Fig. 1. Comparison of results (using 5 parameters).

From Table 1, it is observed that the accuracy is of the order nearly $10^{-5}$ using five parameters in the case of using Bernstein polynomials.

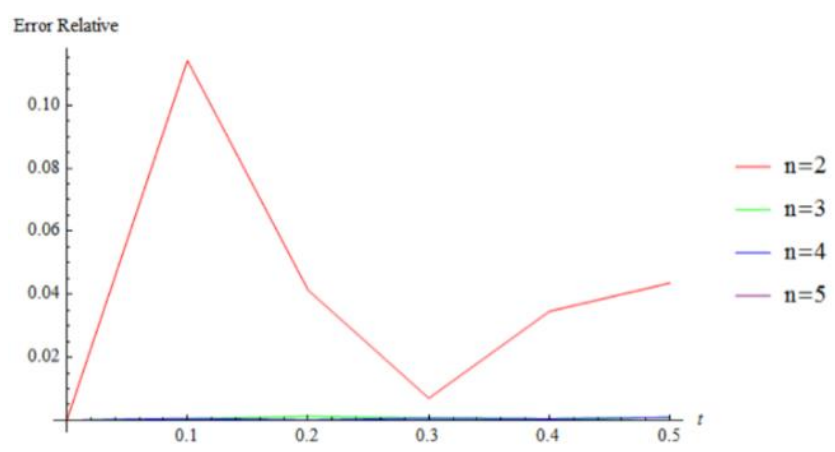

Fig. 2a. Error function when Bernstein polynomial is used

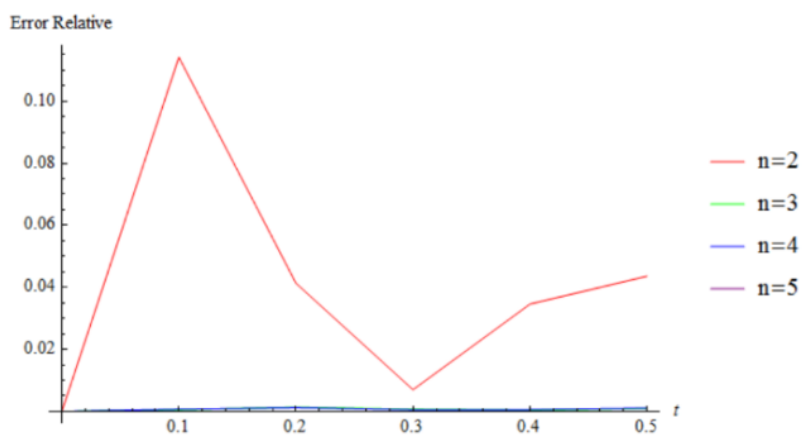

Fig. 2b. Error function when Bernoulli polynomial is used

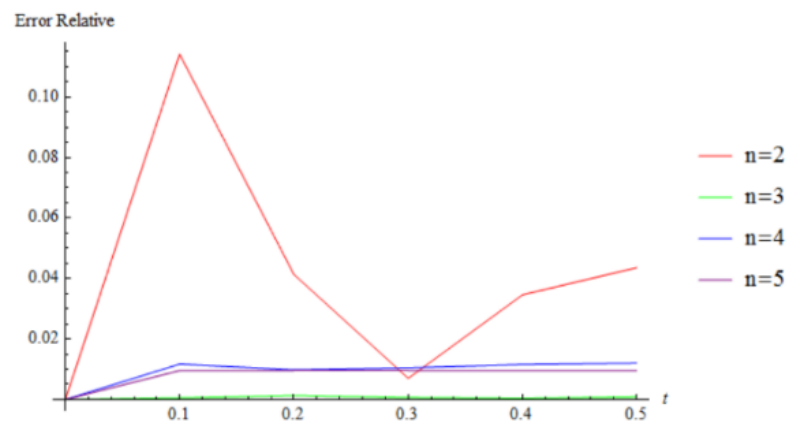

Fig. 2c. Error function when Legendre polynomial is used 


\section{Example 2}

Consider the one-dimensional heat equation ${ }^{4}$

$$
u_{t}=u_{x x} ; 0<x<2 ; t>0
$$

with boundary conditions: $u(0, t)=u(2, t)=0$

and initial conditions:

$$
\begin{aligned}
& u(x, 0)=u_{0}(x)=10 x ; 0 \leq x \leq 1 \\
& u(x, 0)=w_{0}(x)=10(2-x) ; \quad 1 \leq x \leq 2
\end{aligned}
$$

The exact solution of this problem is obtained by using a well-known analytical method is given by

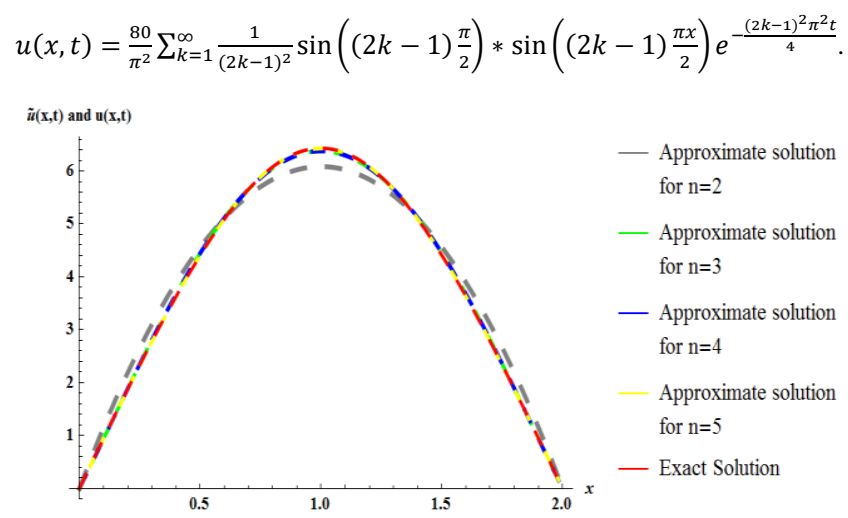

Fig. 3. Comparison of results

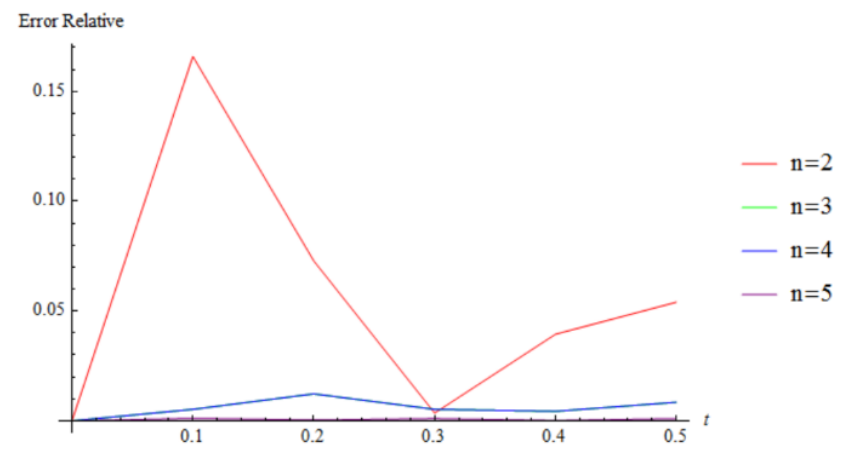

Fig. 4. Error function for Bernstein polynomials

\section{Geometrical Interpretation of Solutions}

If we consider the heat conduction problem mentioned in example 2 for a typical metal bar, then we can say that at the two ends of the bar, i.e. at $x=0$ and $x=2$ the temperature is zero i.e. $u(x, t)=0$. Also for a fixed length of the rod, $x=x_{0}$ the temperature in the rod decreases as $t$ increases. It indicates that the temperature is very high at the beginning and as $t \rightarrow \infty$, gradually the temperature decreases and eventually it becomes zero. Here the center of the $\operatorname{rod}(x=1)$ is the line of symmetry and at any time we get the maximum temperature is at the center. It is also noticeable that for $t=0$ the temperature is discontinuous at the two ends. For different number of Bernstein polynomials and the corresponding graphs of error functions are shown in Fig.3 and Fig.4, respectively. Also the existing results and the results obtained the proposed method with relative errors are displayed in Table 2.
For simplicity, we show the temperature behavior of the bar graphically at different times using our obtained approximate solutions (using 5 parameter Bernstein polynomials) in Fig. 5.

\begin{tabular}{|c|c|c|c|c|}
\hline \multirow[t]{2}{*}{$x$} & \multirow[t]{2}{*}{$\begin{array}{l}\text { Exact } \\
\text { Solution }\end{array}$} & \multirow{2}{*}{$\begin{array}{l}\text { Refer } \\
\text { Results } \\
{[4]}\end{array}$} & $\begin{array}{l}\text { Approximate } \\
\text { Solution }\end{array}$ & Relative Error \\
\hline & & & \multicolumn{2}{|c|}{ Parameter used $n=2$} \\
\hline 0.2 & 1.878702795 & 1.87919 & 2.190377202 & $1.6589 \times 10^{-1}$ \\
\hline 0.4 & 3.629673099 & 3.63355 & 3.894003915 & $7.2825 \times 10^{-2}$ \\
\hline 0.6 & 5.092888161 & 5.10390 & 5.110880139 & $3.5328 \times 10^{-3}$ \\
\hline 0.8 & 6.080815118 & 6.09979 & 5.841005873 & $3.9437 \times 10^{-2}$ \\
\hline \multirow[t]{2}{*}{1.0} & 6.431765995 & 6.45430 & 6.084381118 & $5.4011 \times 10^{-2}$ \\
\hline & & & \multicolumn{2}{|c|}{ Parameter used $n=3$} \\
\hline 0.2 & 1.878702795 & 1.87919 & 1.888517509 & $5.2241 \times 10^{-3}$ \\
\hline 0.4 & 3.629673099 & 3.63355 & 3.674247885 & $1.2281 \times 10^{-2}$ \\
\hline 0.6 & 5.092888161 & 5.10390 & 5.119528559 & $5.2309 \times 10^{-3}$ \\
\hline 0.8 & 6.080815118 & 6.09979 & 6.054600554 & $4.3110 \times 10^{-3}$ \\
\hline \multirow[t]{2}{*}{ 1. $C$} & 6.431765995 & 6.45430 & 6.377608485 & $8.4203 \times 10^{-3}$ \\
\hline & & & \multicolumn{2}{|c|}{ Parameter used $n=4$} \\
\hline 0.2 & 1.878702795 & 1.87919 & 1.888487582 & $5.2083 \times 10^{-3}$ \\
\hline 0.4 & 3.629673099 & 3.63355 & 3.674190851 & $1.2265 \times 10^{-2}$ \\
\hline 0.6 & 5.092888161 & 5.10390 & 5.119450112 & $5.2155 \times 10^{-3}$ \\
\hline 0.8 & 6.080815118 & 6.09979 & 6.054508439 & $4.3262 \times 10^{-3}$ \\
\hline \multirow[t]{2}{*}{1.0} & 6.431765995 & 6.45430 & 6.377511676 & $8.4354 \times 10^{-3}$ \\
\hline & & & \multicolumn{2}{|c|}{ Parameter used $n=5$} \\
\hline 0.2 & 1.878702795 & 1.87919 & 1.876559485 & $1.1408 \times 10^{-3}$ \\
\hline 0.4 & 3.629673099 & 3.63355 & 3.631102669 & $3.9386 \times 10^{-4}$ \\
\hline 0.6 & 5.092888161 & 5.10390 & 5.098107775 & $1.0249 \times 10^{-3}$ \\
\hline$\overline{0.8}$ & 6.080815118 & 6.09979 & 6.079736517 & $1.7738 \times 10^{-4}$ \\
\hline 1. 0 & 6.431765995 & 6.45430 & 6.425442547 & $9.8316 \times 10^{-4}$ \\
\hline 4 & 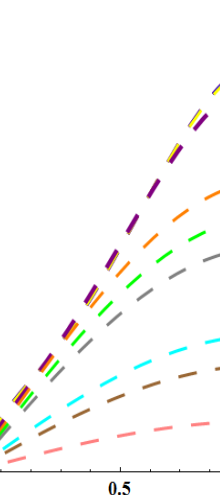 & - & & $\begin{aligned}-\mathrm{t} & =0.00001 \\
-\mathrm{t} & =0.0001 \\
-\mathrm{t} & =0.001 \\
-\mathrm{t} & =0.01 \\
-\mathrm{t} & =0.1 \\
-\mathrm{t} & =0.15 \\
-\mathrm{t} & =0.2 \\
-\mathrm{t} & =0.4 \\
-\mathrm{t} & =0.5 \\
-\mathrm{t} & =0.8\end{aligned}$ \\
\hline
\end{tabular}

Table 2. Comparison of results using Bernstein Polynomials (at $t=0.1$ )

Fig. 5. Temperature profile for different time values. 


\section{Formulation of One Dimensional Wave Equation}

Here we consider a simple model problem in one dimension hyperbolic PDE. Approximating a 1-D wave equation is nothing but solving an IBVP. Let us consider the standard form of IBVP of the form:

$$
\begin{array}{r}
\rho(x) \frac{\partial^{2} u(x, t)}{\partial t^{2}}+\mu(x) \frac{\partial u(x, t)}{\partial t}-\frac{\partial}{\partial x}\left(\alpha(x) \frac{\partial u(x, t)}{\partial x}\right)+ \\
\beta(x) u(x, t)=f(x, t) ; a<x<b ; t>t_{0}
\end{array}
$$

with boundary Conditions:

at $x=a\left(t>t_{0}\right)$,

$u(a, t)=u_{a}(t),\left(-\alpha(x) \frac{\partial u(x, t)}{\partial x}\right)_{a}=\tau_{a}(t) ;$

at $x=b\left(t>t_{0}\right)$,

$u(b, t)=u_{b}(t), \quad\left(-\alpha(x) \frac{\partial u(x, t)}{\partial x}\right)_{b}=\tau_{b}(t)$

and the initial conditions at $t=t_{0}(a<x<b)$

$u\left(x, t_{0}\right)=u_{0}(x),\left(\frac{\partial u(x, t)}{\partial t}\right)_{t_{0}}=v_{0}(x)$.

In the above equation $\rho$ is a density (mass/volume) and $\rho(x) \frac{\partial^{2} u(x, t)}{\partial t^{2}}$ is an inertial force (per volume). Also $\mu$ represents viscosity and $\mu(x) \frac{\partial u(x, t)}{\partial t}$ is known as the damping force.

Let the trial solution of Eq. (7) be of the form

$\tilde{u}(x, t)=\sum_{j=1}^{n} a_{j}(t) \varphi_{j}(x)$

Then the Galerkin Weighted Residual equation for the interval $a<x<b$ may be written as

$$
\begin{gathered}
\int_{a}^{b}\left[\rho(x) \frac{\partial^{2} \tilde{u}(x, t)}{\partial t^{2}}+\mu(x) \frac{\partial \tilde{u}(x, t)}{\partial t}-\frac{\partial}{\partial x}\left(\alpha(x) \frac{\partial \tilde{u}(x, t)}{\partial x}\right)\right. \\
+\beta(x) \tilde{u}(x, t)-f(x, t)] \varphi_{i}(x) d x=0
\end{gathered}
$$

or,

$$
\begin{aligned}
\sum_{j=1}^{n} M_{i, j} \frac{d^{2} a_{j}(t)}{d t^{2}}+\sum_{j=1}^{n} C_{i, j} \frac{d a_{j}(t)}{d t}+ \\
\sum_{j=1}^{n} K_{i, j} a_{j}(t)=F_{i}(t) ; i=1,2,3, \ldots, n
\end{aligned}
$$

which can be expressed in usual matrix notation as

$[M]\left\{\frac{d^{2} a(t)}{d t^{2}}\right\}+[C]\left\{\frac{d a(t)}{d t}\right\}+[K]\{a(t)\}=\{F(t)\}$

where,

$$
\begin{aligned}
& {[M]=M_{i, j}=\int_{a}^{b} \varphi_{i}(x) \rho(x) \varphi_{j}(x) d x} \\
& \begin{aligned}
& {[C]=C_{i, j}=} \int_{a}^{b} \varphi_{i}(x) \mu(x) \varphi_{j}(x) d x \\
& {[K]=K_{i, j}=} \int_{a}^{b} \frac{d \varphi_{i}(x)}{d x} \alpha(x) \frac{d \varphi_{j}(x)}{d x} d x \\
&+\int_{a}^{b} \varphi_{i}(x) \beta(x) \varphi_{j}(x) d x \\
& F_{i}=\int_{a}^{b} f(x, t) \varphi_{i}(x) d x-\left[\left(-\alpha(x) \frac{\partial \widetilde{u}(x, t)}{\partial x}\right) \varphi_{i}(x)\right]_{a}^{b}
\end{aligned}
\end{aligned}
$$

Such equations usually arise in structural dynamics, where $[M]$ denotes the mass matrix, $[C]$ is the damping matrix, and $[K]$ is the stiffness matrix, whereas $F_{i}$ is called the load matrix. The damping matrix $[C]$ is often taken to be a linear combination of the mass and stiffness matrices that is $[C]=\beta_{1}[M]+\beta_{2}[K]$, where $\beta_{1}$ and $\beta_{2}$ are determined from physical experiments. In the present study, we will not consider damping (i.e. $[C]=[0]$ ) in the numerical examples, although the theoretical developments will account for it.

Now, it is the only requirement for us to solve Eq. (8) using the initial condition. Since we have considered the damping matrix $[C]$ is zero. Hence from the application of weighted residual method we get a constrained system of ordinary differential equations. This system of differential equations (9) will be treated analytically by decomposing the general solution $a(t)$ into a homogeneous solution $a_{h}$ and a particular solution $a_{p}$ according to $a(t)=a_{h}(t)+$ $a_{p}(t)$. Once we get the values of $a(t)$, substituting them into Eq. (8), we will get our desired approximate solution of the PDE (7).

\section{Example 3}

Consider the one-dimensional linear wave equation ${ }^{3}$

$$
u_{t t}=u_{x x} ; \quad 0<x<1 ; t>0
$$

with boundary conditions: $u(0, t)=u(1, t)=0$ and initial conditions:

$u(x, 0)=u_{0}(x)=x(1-x), u_{t}(x, 0)=v_{0}(x)=0$.

The exact solution of this problem is obtained by using a well-known analytical method:

$$
\begin{array}{r}
u(x, t)=\frac{8}{\pi^{3}} \sum_{k=1}^{\infty} \frac{1}{(2 k-1)^{3}} \cos ((2 k-1) \pi t) * \\
\sin ((2 k-1) \pi x) .
\end{array}
$$

\section{Tabular Analysis}

Since we make a comparison table for the purpose of displaying the efficiency of using piecewise polynomials. By the help of these polynomials we get our desired solutions more quickly. Also summarizing the Table 3 we can see that the approximation is same for two parameters. But if we add more parameters we get slightly better approximation. From our computational point of view it is undoubtedly true that using Bernstein polynomials is the best choice in WRM for faster convergence. Bernoulli polynomials also generate good approximation while Legendre polynomials is less accurate.

From Table 3, it is also observed that the accuracy is of the order nearly $10^{-3}$ has been found using only 3,4 or 5 parameters. Thus we may note that if we use few number of polynomials then the result is stable and for large number of polynomials the result may be unstable. Fig. 6 shows the comparison of approximation using different types of polynomials and the corresponding relative errors are also depicted in Fig.7, respectively. 
Table 3. Comparison of results at various points of the domain (for $t=0.2$ )

\begin{tabular}{|c|c|c|c|c|}
\hline \multirow{3}{*}{$x$} & \multirow{3}{*}{$\begin{array}{l}\text { Exact } \\
\text { Solutions }\end{array}$} & \multicolumn{3}{|l|}{ Relative Error } \\
\hline & & $\begin{array}{l}\text { Bernstein } \\
\text { Polynomials }\end{array}$ & $\begin{array}{l}\text { Bernoulli } \\
\text { polynomials }\end{array}$ & $\begin{array}{l}\text { Legendre } \\
\text { Polynomials }\end{array}$ \\
\hline & & \multicolumn{3}{|c|}{ Parameter used $n=2$} \\
\hline 0.1 & 0.0600000001 & $2.0987 \times 10^{-1}$ & $2.0987 \times 10^{-1}$ & $2.0987 \times 10^{-1}$ \\
\hline 0.2 & 0.1199999999 & $7.5438 \times 10^{-2}$ & $7.5438 \times 10^{-2}$ & $7.5438 \times 10^{-2}$ \\
\hline 0.3 & 0.1699999997 & $3.6384 \times 10^{-3}$ & $3.6384 \times 10^{-3}$ & $3.6384 \times 10^{-3}$ \\
\hline 0.4 & 0.1999999998 & $3.2106 \times 10^{-2}$ & $3.2106 \times 10^{-2}$ & $3.2106 \times 10^{-2}$ \\
\hline \multirow[t]{2}{*}{0.5} & 0.2099999998 & $3.9788 \times 10^{-2}$ & $3.9788 \times 10^{-2}$ & $3.9788 \times 10^{-2}$ \\
\hline & & \multicolumn{3}{|c|}{ Parameter used $n=3$} \\
\hline 0.1 & 0.0600000001 & $3.6745 \times 10^{-4}$ & $3.2339 \times 10^{-4}$ & $3.2248 \times 10^{-4}$ \\
\hline 0.2 & 0.1199999999 & $5.6705 \times 10^{-3}$ & $5.6587 \times 10^{-3}$ & $5.6573 \times 10^{-3}$ \\
\hline 0.3 & 0.1699999997 & $8.7900 \times 10^{-3}$ & $8.7971 \times 10^{-3}$ & $8.7953 \times 10^{-3}$ \\
\hline 0.4 & 0.1999999998 & $3.6856 \times 10^{-3}$ & $3.6682 \times 10^{-3}$ & $3.6705 \times 10^{-3}$ \\
\hline \multirow[t]{2}{*}{0.5} & 0.2099999998 & $9.2106 \times 10^{-3}$ & $9.1898 \times 10^{-3}$ & $9.1925 \times 10^{-3}$ \\
\hline & & \multicolumn{3}{|c|}{ Parameter used $n=4$} \\
\hline 0.1 & 0.0600000001 & $3.4569 \times 10^{-4}$ & $5.6860 \times 10^{-4}$ & $8.1867 \times 10^{-3}$ \\
\hline 0.2 & 0.1199999999 & $5.6764 \times 10^{-3}$ & $5.8933 \times 10^{-3}$ & $1.6792 \times 10^{-3}$ \\
\hline 0.3 & 0.1699999997 & $8.8108 \times 10^{-3}$ & $9.0254 \times 10^{-3}$ & $2.1487 \times 10^{-3}$ \\
\hline 0.4 & 0.1999999998 & $3.6576 \times 10^{-3}$ & $3.4399 \times 10^{-3}$ & $1.0039 \times 10^{-2}$ \\
\hline \multirow[t]{2}{*}{0.5} & 0.2099999998 & $9.1816 \times 10^{-3}$ & $8.9613 \times 10^{-3}$ & $1.5481 \times 10^{-2}$ \\
\hline & & \multicolumn{3}{|c|}{ Parameter used $n=5$} \\
\hline 0.1 & 0.0600000001 & $5.0952 \times 10^{-2}$ & $2.7977 \times 10^{-3}$ & $1.3001 \times 10^{-2}$ \\
\hline 0.2 & 0.1199999999 & $3.1013 \times 10^{-2}$ & $4.3409 \times 10^{-3}$ & $1.4018 \times 10^{-2}$ \\
\hline 0.3 & 0.1699999997 & $9.5524 \times 10^{-3}$ & $4.9233 \times 10^{-3}$ & $4.5584 \times 10^{-3}$ \\
\hline 0.4 & 0.1999999998 & $7.9343 \times 10^{-3}$ & $3.3672 \times 10^{-4}$ & $9.8934 \times 10^{-3}$ \\
\hline 0.5 & 0.2099999998 & $6.5271 \times 10^{-3}$ & $2.8852 \times 10^{-3}$ & $1.2488 \times 10^{-2}$ \\
\hline
\end{tabular}

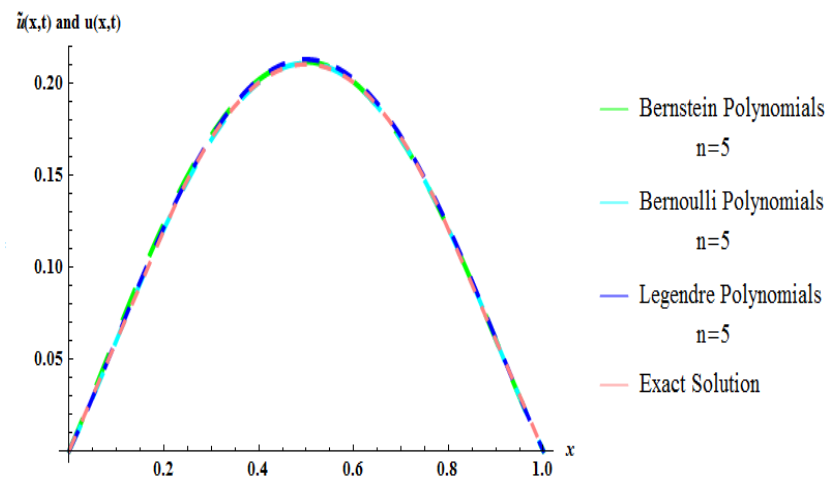

Fig. 6. Comparison of results (using 5 parameter)

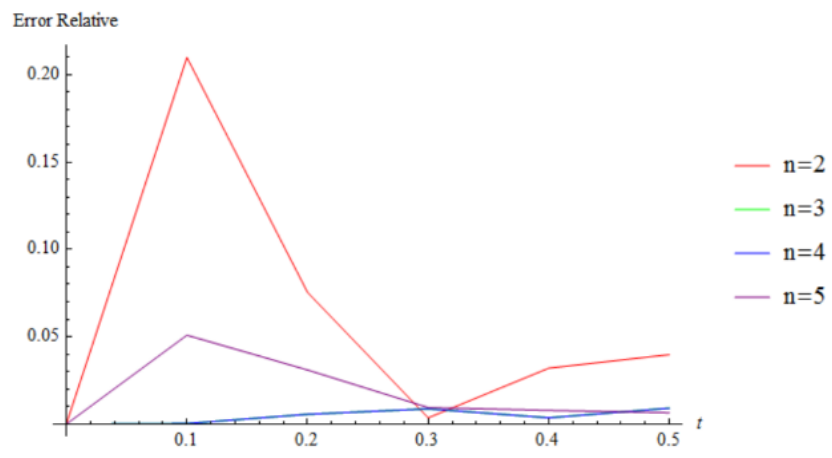

Fig. 7a. Error function when Bernstein polynomial is used

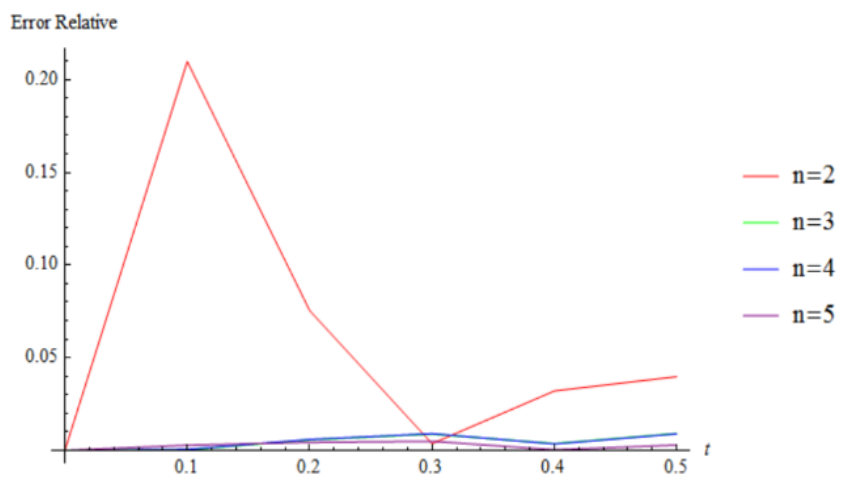

Fig. 7b. Error function when Bernoulli polynomial is used

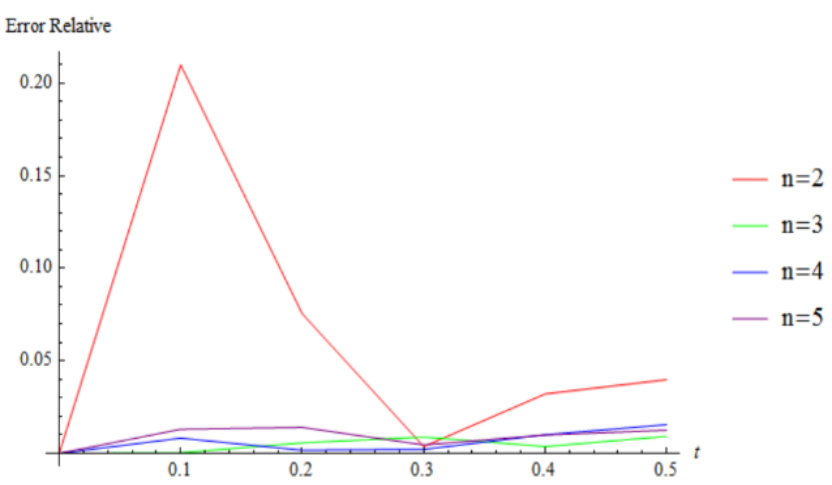

Fig. 7c. Error function when Legendre polynomial is used

Example 4

Consider the one dimensional equation of a plucked spring ${ }^{5}$

$u_{t t}=u_{x x} ; 0<x<2 ; t>0$

with boundary conditions: $u(0, t)=u(2, t)=0$

and initial conditions:

$$
\begin{aligned}
u(x, 0)=u_{0}(x)= & 0.1 x ; \quad 0 \leq x \leq 1 \\
u(x, 0)=w_{0}(x)= & 0.1(2-x) ; 1 \leq x \leq 2 \\
& u_{t}(x, 0)=v_{0}(x)=0
\end{aligned}
$$

The exact solution is:

$u(x, t)=\frac{0.8}{\pi^{2}} \sum_{k=1}^{\infty} \frac{1}{k^{2}} \sin \left(\frac{k \pi}{2}\right) \sin \left(\frac{k \pi}{2} x\right) \cos \left(\frac{k \pi}{2} t\right)$.

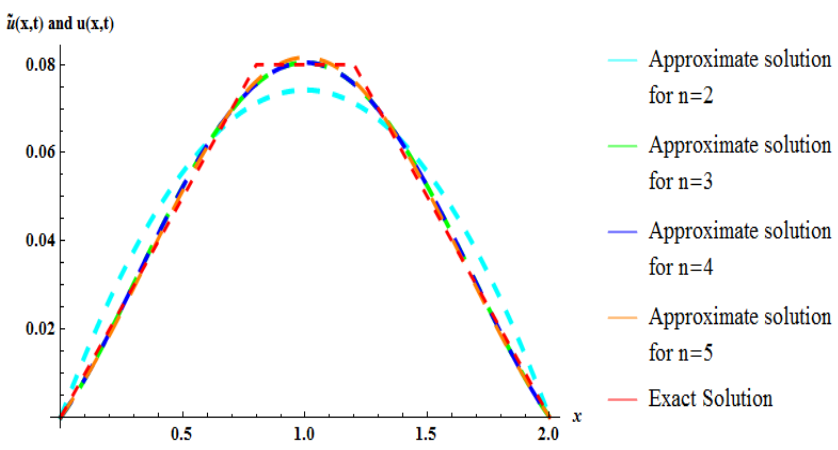

Fig. 8. Comparison of results 
Table 4. Comparison of results (at $t=0.2$ )

\begin{tabular}{|c|l|l|l|}
\hline \multirow{2}{*}{$\boldsymbol{x}$} & \multirow{2}{*}{$\begin{array}{l}\text { Reference } \\
\text { Results [5] }\end{array}$} & $\begin{array}{l}\text { Approximate } \\
\text { Solution }\end{array}$ & Relative Error \\
\cline { 3 - 4 } & & Parameter used $n=2$ \\
\hline $\mathbf{0 . 2}$ & 0.0199999997 & 0.0267304298 & $3.3652 \times 10^{-1}$ \\
\hline $\mathbf{0 . 4}$ & 0.0399999991 & 0.0475207640 & $1.8802 \times 10^{-1}$ \\
\hline $\mathbf{0 . 6}$ & 0.0599999965 & 0.0623710028 & $3.9517 \times 10^{-2}$ \\
\hline $\mathbf{0 . 8}$ & 0.0799594708 & 0.0712811460 & $1.0853 \times 10^{-1}$ \\
\hline $\mathbf{1 . 0}$ & 0.0799999935 & 0.0742511938 & $7.1860 \times 10^{-2}$ \\
\hline & & Parameters used $n=3$ \\
\hline $\mathbf{0 . 2}$ & 0.0199999997 & 0.0192095464 & $3.9523 \times 10^{-2}$ \\
\hline $\mathbf{0 . 4}$ & 0.0399999991 & 0.0417434869 & $4.3587 \times 10^{-2}$ \\
\hline $\mathbf{0 . 6}$ & 0.0599999965 & 0.0619069348 & $3.1782 \times 10^{-2}$ \\
\hline $\mathbf{0 . 8}$ & 0.0799594708 & 0.0756321141 & $5.4119 \times 10^{-2}$ \\
\hline $\mathbf{1 . 0}$ & 0.0799999935 & 0.0804783589 & $5.9796 \times 10^{-3}$ \\
\hline & & Parameters used $n=4$ \\
\hline $\mathbf{0 . 2}$ & 0.0199999997 & 0.0192093678 & $3.9532 \times 10^{-2}$ \\
\hline $\mathbf{0 . 4}$ & 0.0399999991 & 0.0417431466 & $4.3579 \times 10^{-2}$ \\
\hline $\mathbf{0 . 6}$ & 0.0599999965 & 0.0619064668 & $3.1775 \times 10^{-2}$ \\
\hline $\mathbf{0 . 8}$ & 0.0799594708 & 0.0756315645 & $5.4126 \times 10^{-2}$ \\
\hline $\mathbf{1 . 0}$ & 0.0799999935 & 0.0804777814 & $5.9723 \times 10^{-3}$ \\
\hline & & Parameters used $n=5$ \\
\hline $\mathbf{0 . 2}$ & 0.0199999997 & 0.0186239202 & $6.8804 \times 10^{-2}$ \\
\hline $\mathbf{0 . 4}$ & 0.0399999991 & 0.0406421817 & $1.6055 \times 10^{-2}$ \\
\hline $\mathbf{0 . 6}$ & 0.0599999965 & 0.0614584727 & $2.4308 \times 10^{-2}$ \\
\hline $\mathbf{0 . 8}$ & 0.0799594708 & 0.0762848119 & $4.5956 \times 10^{-2}$ \\
\hline $\mathbf{1 . 0}$ & 0.0799999935 & 0.0816464505 & $2.0581 \times 10^{-2}$ \\
\hline & & \multicolumn{2}{|l}{} \\
\hline
\end{tabular}

Physical Interpretation of Solutions

If we consider the hyperbolic problem mentioned in example 4 for a typical plucked spring, then we can say that at the two ends of the spring, i.e. at $x=0$ and $x=2$ the spring displacement is zero i.e. $u(x, t)=0$.

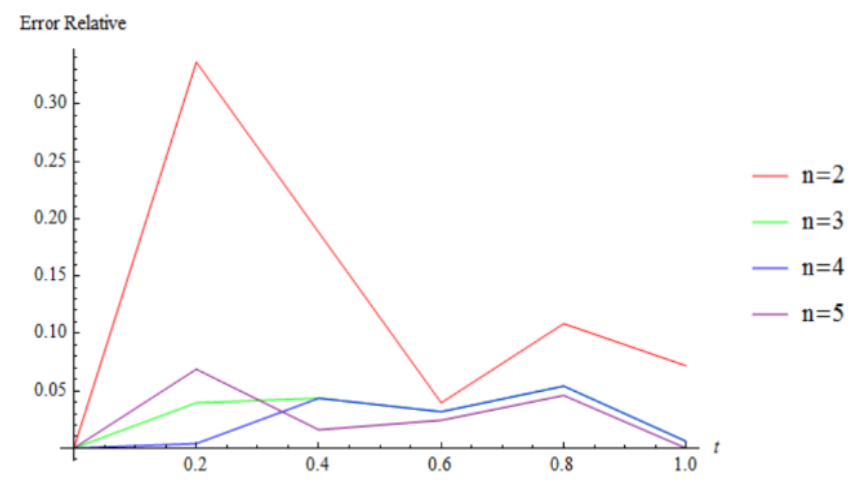

Fig. 9. Error function for Bernstein polynomial

Notice that unlike the heat equation the solution curve does not become smoother, the sharp edges remain. For each fixed $t$, we can think of the function $u(x, t)$ is just a function of spatial coordinate $x$. This function also gives us the shape of the spring at any time $t$. In this case we use only Bernstein polynomial in different numbers and the approximations and relative errors are shown in Fig. 8 and Fig. 9, respectively. The existing ${ }^{5}$ solutions and the results obtained using the proposed method in this paper are summarized in Table 4. From the Table 4, we may comment that if we use large number of Bernstein polynomials then the result may be unstable. For simplicity, we also show the shape of the spring graphically at different times using our obtained approximate solutions (using 5 Bernstein Polynomials) in Fig. 10.

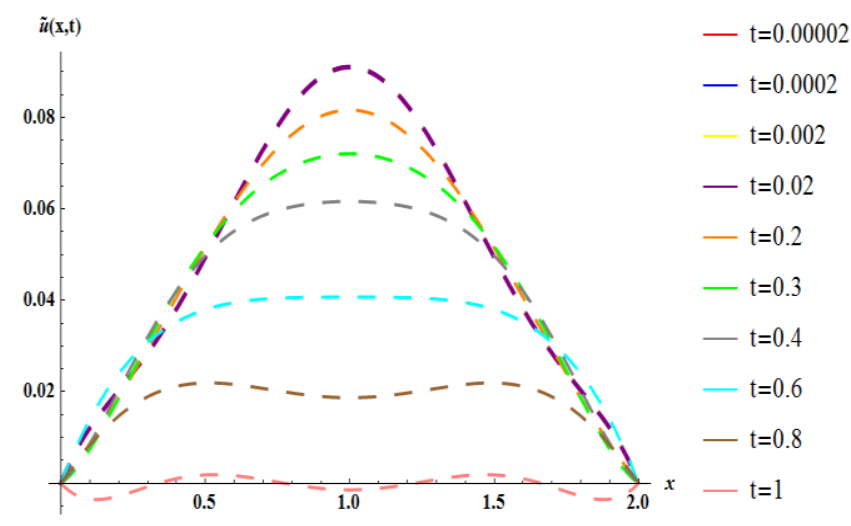

Fig. 10. Graphical representation of spring displacement profile for different time values.

\section{Conclusions}

In this paper, we have proposed Galerkin weighted residual method to solve one dimensional parabolic and hyperbolic PDEs numerically. In this method we have used piecewise polynomials such as: Bernstein, Bernoulli and Legendre as trial functions. A rigorous matrix formulation has been derived. Several numerical examples have been tested to verify the stability, consistency, and convergence of the proposed scheme. The computed approximate solutions are then compared with exact solutions and we have found a good agreement. The approximate solutions converge to the exact solutions with large significant digits even though only a few number of polynomials have been used. We may conclude that our proposed method can be applied to higher order IBVPs to get desired accuracy.

\section{Acknowledgement}

The authors are thankful to the reviewer(s) and editorial board for their comments and suggestions for improvement of the quality of the first version of this manuscript. Also the first author is grateful to the National Science and Technology (NST), Ministry of Science and Technology, Govt. of People's Republic of Bangladesh, for granting the "NST Fellowship" during the period of research work. 


\section{References}

1. David. S. Burnett, 1988. Finite Element Analysis, From Concepts to Applications, Addison-Wesley.

2. Bickford, W. B., 1994. A First course in the Finite Element Method, Second Edition.

3. Reddy, J. N., 1986. Applied Functional Analysis and Variational Methods in Engineering, McGraw-Hill, Illustrated Edition.

4. Zhong, W., Z. Jianing, X. X. Zhong, 1996. On a new time integration method for solving time dependent partial differential equations, Comput. Methods Appl. Mech. Engrg. 130, 163 - 178.

5. Jiri Lebl, 2012. Notes on Diffy Qs: Differential Equations for Engineers.

6. Islam, Md. Shafiqul, Afroza Shirin, 2010. Numerical solutions of Fredholm integral equations of second kind using piecewise Bernoulli polynomials, The Dhaka University Journal of Science, 58 (2), 264 - 272.

7. Islam, Md. Shafiqul, Md. Bellal Hossain, 2015. Numerical Solutions of Eighth Order BVP by the Galerkin Residual Technique with Bernstein and Legendre Polynomials, Applied Mathematics and Computation, 261, 48 - 59. 
